УДК 681.513 .5

\title{
APPLICATION OF LINEAR MATRIX INEQUALITIES DURING SYNTHESIS OF MODULAR CONTROL BY MULTIDIMENSIONAL LINEAR SYSTEMS
}

\author{
O. Lobok, B. Goncharenko \\ National University of Food Technologies \\ M. Sych \\ National University of Bioresources and Natural Resources of Ukraine
}

$\quad$ Key words:
Dynamical system
Modal control
Regulators
D-stability
Luenberger observers
Linear matrix inequalities
Kroneker product of
matrices

Article history:

Received 14.05.2018

Received in revised form

31.05.2018

Accepted 19.06.2018

Corresponding author:

B. Goncharenko

E-mail:

GoncharenkoBN@i.ua

\begin{abstract}
The paper gives a solution to the problem of constructing modal regulators for linear multidimensional systems that provide D-stability (asymptotic stability) of the control object. The control is represented as regulators providing feedback on the output of the control object, and it uses the full and low order observers of Luenberger. To calculate the matrices of the regulators, we use the technique of linear matrix inequalities and generalization of the Lyapunov stability concept ( $D$-stability). The theorems which give necessary and sufficient conditions for $D$-stability of the controlled system are given.
\end{abstract}

The constructive solution of the problem of synthesis of $D$-stabilizing (modal) regulators according to the measured output of the control object, based on the construction of observers of the state of the object of the complete and reduced order, is given. The solution is obtained based on the use of the theory of linear matrix inequalities (LMI). For numerical simulation of the obtained modal regulators you can use effective methods of convex optimization and corresponding software that is included in a number of application packages, in particular, in the MatLab system.

In this paper we describe methods for solving not only the direct problem of modal control, when the choice of parameters of a regulator is ensured by the coincidence of the roots of the characteristic equation of a closed system with a predefined set of complex numbers located on the left side of the complex plane, but also other problems of modal control, in which the requirement of the exact placement of the roots in the left integrated half-plane is not superimposed, but only their membership in certain specified areas is required. Such areas, described by a system of linear matrix inequalities (LMI), are called LMI domains.

DOI: $10.24263 / 2225-2924-2018-24-3-4$ 


\section{ЗАСТОСУВАННЯ ЛІНІЙНИХ МАТРИЧНИХ НЕРІВНОСТЕЙ ПРИ СИНТЕЗІ МОДАЛЬНОГО КЕРУВАННЯ БАГАТОМІРНИМИ ЛІНІЙНИМИ СИСТЕМАМИ}

\section{О.П. Лобок, Б.М. Гончаренко}

Наиіональний університет харчових технологій М.А. Сич

Національний університет біоресурсів і природокористування України

У статті наведено розв'язок задачі побудови модальних регуляторів для лінійних багатовимірних систем, що забезпечують D-стійкість (асимптотичну стійкість) об’єкта керування. Керування представлено у вигляді регуляторів, що забезпечують зворотний зв'язок за виходом об'єкта керування, $i$ здійснюється з використанням спостерігачів Луенбергера повного $i$ зниженого порядку. Для обчислення матрищь регуляторів застосовано техніку лінійних матричних нерівностей $i$ узагальнення поняття стійкості за Ляпуновим (D-стійкість). Наведено теореми, щчо дають необхідні і достатні умови D-стійкості керованої системи.

Запропоновано конструктивний розв'язок задачі синтезу D-стабілізувальних (модальних) регуляторів за вимірюваним виходом об 'єкта керування, заснований на побудові спостерігачів стану об'єкта певного порядку. Розв'язок отримано на основі використання теорії лінійних матричних нерівностей (LMI). Для чисельного моделювання отриманих модальних регуляторів можна використовувати ефективні методи опуклої оптимізації і відповідне програмне забезпечення, яке входить до ряду пакетів прикладних програм, зокрема, в систему MatLab.

Описано методи розв'язання не тільки прямої задачі модального керування, коли вибір параметрів регулятора забезпечує збіг коренів характеристичного рівняння замкненої системи з попередньо заданим набором комплексних чисел, розташованих у лівій частині комплексної площини, але й інших задач модального регулювання, в яких вимога точного розміщення коренів у лівій комплексній півплощині вже не накладається, а потрібна лише їх приналежність до заданих областей. Такі області, описані системою лінійних матричних нерівностей, називаються LMI-областями.

Ключові слова: динамічна система, модальне керування, регулятори, D-стійкість, спостерігачі Луенбергера, лінійні матричні нерівності, кронекеровий добуток матриць.

Постановка проблеми. Часто в задачах керування з численної множини стабілізувальних керувань потрібно виділити певну підмножину, що забезпечує для системи додаткові властивості. Такою властивістю може бути, наприклад, розташування коренів характеристичного многочлена замкнутої системи в заданій області комплексної площини. Керування, що має такі 
додаткові властивості, називають модальним керуванням, а регулятор, що забезпечує його, вважають модальним. Модальне керування відноситься до кореневих методів синтезу лінійних САУ, при яких, виходячи з бажаних показників якості керування, будується бажаний характеристичний поліном, а отже, визначається місце розташування коренів характеристичного рівняння. Характерні значення коренів латиною називаються модами, звідси назва регулятора і керування — модальні.

Можлива така постановка задачі: вибором параметрів керування забезпечити точний збіг коренів характеристичного рівняння замкненої системи з наперед заданим набором комплексних чисел, розташованих у лівій частині (умова стійкості) комплексної площини. Таку задачу іноді називають прямою задачею модального керування. У пропонованій статті описуються такі методи розв'язання інших завдань модального регулювання, в яких вимога точного розміщення коренів у лівій комплексної півплощині вже не накладається, а потрібна лише їх приналежність до заданого домену.

Як зазначалося вище, задача модального керування пов'язана з побудовою регулятора, при якому полюси замкненої системи розташовуються в заданих точках або в заданих областях комплексної площини. Значення таких характеристик замкнутої системи, як час перехідного процесу, демпфірування, швидкість перехідних процесів у регуляторі та інших визначаються розташуванням власних значень матриці замкненої системи в певних областях комплексної площини.

Мета статті: розглянути задачі модального керування щодо таких областей, які можуть бути описані системою лінійних матричних нерівностей - LMIобластями. Показати, що до цих областей відносяться вертикальні і горизонтальні смуги, кола, конічні сектори, а також перетини таких областей.

Викладення основних результатів дослідження. Один 3 ефективних способів розв'язання задач синтезу модального керування пов'язаний із застосуванням квадратичних функцій Ляпунова і техніки лінійних матричних нерівностей.

Загальний підхід до синтезу модального керування заснований на використанні LMI. Виявляється, що області певного виду на комплексній площині, в яких потрібно розмістити власні значення матриці замкненої лінійної системи, можна описати лінійними матричними нерівностями, тобто як LMI-області [1; 3 -5], спочатку записуючи необхідні нерівності щодо змінних $x=\operatorname{Re}(z)$ i $y=\operatorname{Im}(z)$, а потім виконуючи їхню заміну на матриці спеціальною підстановкою.

Розглянемо в загальному випадку формальну процедуру отримання лінійних матричних нерівностей, що визначають критерії розміщення всіх власних чисел матриці об'єкта керування в необхідній LMI-області. Зауважимо, що для чисельного розв'язування отриманих лінійних матричних нерівностей можуть бути використані існуючі ефективні алгоритми, які реалізовані в математичних пакетах, зокрема в пакеті MatLab $[1 ; 8]$.

Оскільки апарат лінійних матричних нерівностей відносно рідко використовується в прикладних задачах керування багатовимірними об'єктами, коротко викладемо основні положення цієї теорії. 
Введемо поняття LMI-області. Нехай $D$ - область лівої комплексної півплощини. Динамічну систему будемо називати $D$-стійкою, якщо всі їі полюси, тобто всі власні значення матриці лежать в області $D$. У цьому випадку матрицю $A$ також будемо називати $D$-стійкою. В окремому випадку, коли $D$ збігається 3 усією лівої комплексної напівплощиною, $D$-стійкість зводиться до асимптотичної стійкості, яка характеризується нерівністю Ляпунова, що є лінійною матричною нерівністю. Тобто матриця $A$ асимптотично стійка тоді і тільки тоді, коли існує симетрична матриця $X$, яка задовольняє нерівності:

$$
A X+X A^{T}<0, X>0 .
$$

Визначимо клас областей, які характеризуються в термінах лінійних матричних нерівностей. Для цього введемо в розгляд матричні функції комплексної змінної $z \in C$ ( $C$ - безліч комплексних чисел), що приймають значення в просторі самопоєднаних ермітових $(m \times m)-$ матриць (Ерміт Шарль, 1822-1901):

$$
f_{D}(z)=P+z G+\bar{z} G^{T},
$$

де $P=P^{T} \in R^{m \times m}$ i $G \in R^{m \times m}$ - задані матриці ( $R^{m \times m}-$ множина дійсних матриць розмірності $m \times m), \bar{z}$ — поєднане комплексне число.

Область

$$
D=\left\{z \in C: f_{D}(z)<0\right\}
$$

називається LMI-областю, породжуваною функцією $f_{D}(z)$, яку часто називають характеристичною функцією області $D$.

3 цього визначення випливає, що LMI-область - це підмножина комплексної площини, яка відображається лінійною матричною нерівністю щодо змінних $x=\operatorname{Re}(z)$ i $y=\operatorname{Im}(z)$. Отже, LMI-області - опуклі. Крім того, позаяк для будь-якого $z \in D$ має місце $f_{D}(\bar{z})=\bar{f}_{D}(z)<0$, то LMI-області симетричні щодо дійсної осі.

Найсуттєвіша властивість LMI-областей полягає в тому, що вони повністю визначаються в термінах лінійних матричних нерівностей щодо симетричної позитивно визначеної матриці. Для того, щоб отримати ці нерівності, поставимо у відповідність до функції $f_{D}(z)$ таку $(m \times m)$ - блочну матрицю:

$$
M(A, X)=P \otimes X+G \otimes(A X)+G^{T} \otimes\left(X A^{T}\right),
$$

де «®»- операція кронекерового добутку матриць (Кронекер Леопольд, $1823-1872)$.

Нагадаємо, що кронекеровим добутком матриць називається блокова матриця, утворена шляхом множення кожного елемента $a_{i j}$ матриці $A$ на матрицю $B$ [2]. 3 огляду на це, зауважимо, що блоки матриці $M(A, X)$ можна записати у вигляді:

$$
M_{i j}(A, X)=p_{i j} X+g_{i j} A X+g_{j i} X A^{T}, i, j=1,2, \ldots, m,
$$

де $p_{i j}, g_{i j}$ - елементи матриць $P$ і $G$ відповідно. 
Для побудови модальних регуляторів, що забезпечують стійкість об'єктів керування, важливим $є$ доведення теореми 1 стійкості [3; 5; 7].

Нехай $D$-LMI - область. Тож матриця $A \in D$-стійкою тоді і тільки тоді, якщо існує матриця $X=X^{T}$, яка задовольняє такі лінійні матричні нерівності:

$$
M(A, X)<0, X>0 .
$$

Якщо матрицю (5) помножити зліва і праворуч на матрицю $E \otimes Y$, де $E-$ одинична матриця, $Y=X^{-1}$, то 3 урахуванням властивостей операції кронекерового добутку після ряду перетворень отримаємо критерій $D$-стійкості матриці $A$ :

$$
L(A, Y)=P \otimes Y+G \otimes(Y A)+G^{T} \otimes\left(A^{T} Y\right)<0, Y=Y^{T}>0 .
$$

На основі теореми 1 можна запропонувати такий алгоритм побудови LMIобластей, що визначають критерій $D$-стійкості системи $\dot{x}(t)=A x(t)$ :

1. Будується характеристична функція $f_{D}(z)$ виду (1), щоб породжувана нею множина $D$ мала потрібний вид.

2. Використовуючи підстановку $(1, z, \bar{z}) \leftrightarrow\left(X, A X, X A^{T}\right)$, функції $f_{D}(z)$ ставиться у відповідність блокова матриця $M(A, X)$ виду (3).

3. Формується система матричних нерівностей виду (5) (або (6)) і знаходиться ії розв'язок щодо матриці $X$ (або $Y$ ).

4. Відповідно до вищенаведеної теореми 1 робиться висновок щодо $D$ стійкості багатовимірної лінійної системи $\dot{x}(t)=A x(t)$.

Відзначимо одну важливу властивість LMI-областей: LMI-області замкнені щодо операції перетину, тобто перетин LMI-областей теж буде LMI-областю.

Розглянемо кілька важливих прикладів побудови LMI-області (рис. 1).

Як перший приклад розглянемо множину $D_{1}=\{z \in C: \operatorname{Re}(z)<-\mu\}$ (рис. 1a), яка відповідає асимптотично стійким системам зі ступенем стійкості, не меншим $\mu$. Очевидно, що цю область породжує функція $f_{D_{1}}(z)=z+\bar{z}+2 \mu, \mathrm{i}$ згідно з теоремою 1 матриця $A \in$ асимптотично стійкою зі ступенем стійкості, не меншим за $\mu$, тоді і тільки тоді, коли існує матриця $X=X^{T}$, яка задовольняє лінійні матричні нерівності виду (5):

$$
A X+X A^{T}+2 \mu X<0, X>0 .
$$

Інший приклад LMI-області $€ D_{2}=\{z \in C:|z+q|<r\}$ - внутрішність кола радіусом $r$ з центром в точці $(-q, 0)$ (рис. 16$)$. Для цієї області

$$
f_{D_{2}}(z)=\left(\begin{array}{cc}
-r & q+z \\
q+\bar{z} & -r
\end{array}\right)<0,
$$

і лінійні матричні нерівності (5), що характеризують цю область, набувають вигляду:

$$
\left(\begin{array}{cc}
-r X & q X+A X \\
q X+X A^{T} & -r X
\end{array}\right)<0, X>0 .
$$



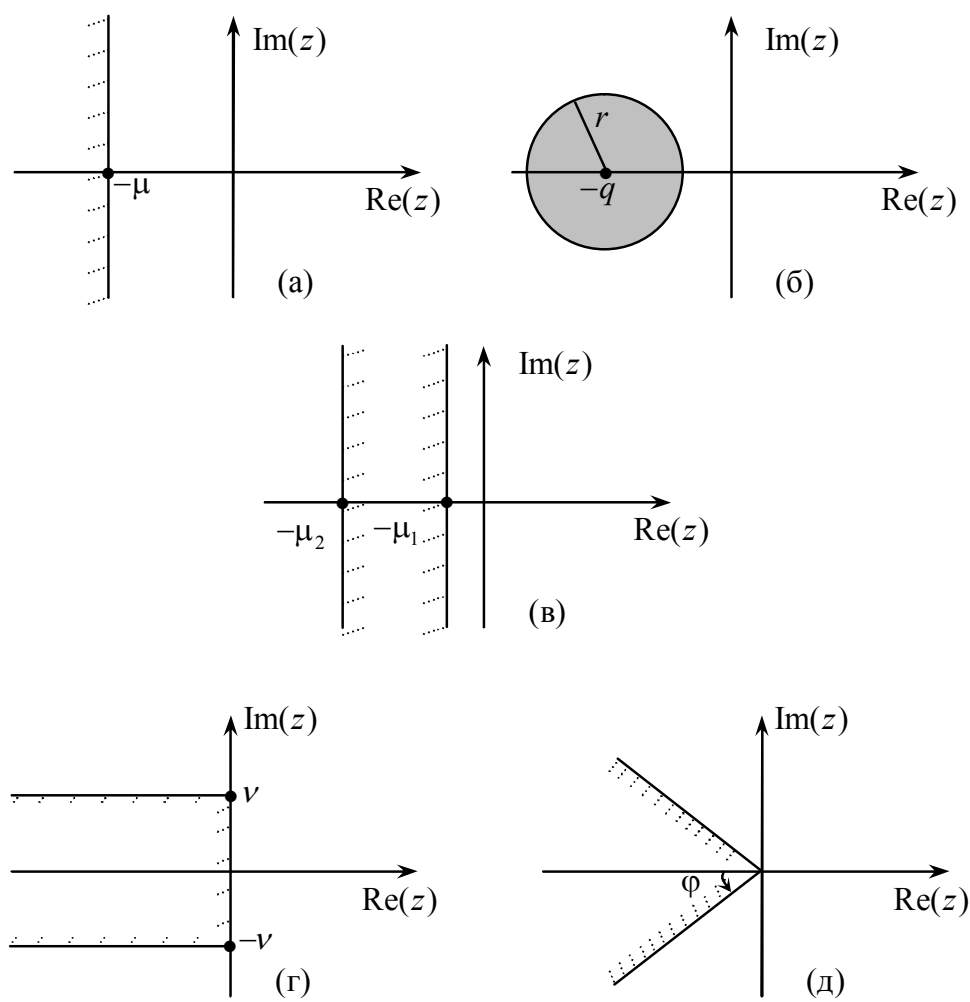

Рис. 1. Приклади LMI-областей стійкості

Вертикальній смузі $D_{3}=\left\{z \in C:-\mu_{2}<\operatorname{Re}(z)<-\mu_{1}\right\}$ (рис. 1в) відповідає функція:

$$
f_{D_{3}}(z)=\left(\begin{array}{cc}
(z+\bar{z})+2 \mu_{1} & 0 \\
0 & -(z+\bar{z})-2 \mu_{2}
\end{array}\right)
$$

i, відповідно, лінійні матричні нерівності:

$$
\left(\begin{array}{cc}
A X+X A^{T}+2 \mu_{1} X & 0 \\
0 & -A X-X A^{T}-2 \mu_{2} X
\end{array}\right)<0, X>0 .
$$

Горизонтальній півсмузі $D_{4}=\{z \in C: \operatorname{Re}(z)<0,-v<\operatorname{Im} z<v\}$ (рис. 1г) відповідає характеристична функція:

$$
f_{D_{4}}(z)=\left(\begin{array}{cc}
-2 v & z-\bar{z} \\
-(z-\bar{z}) & -2 v
\end{array}\right)
$$

і лінійні матричні нерівності:

$$
\left(\begin{array}{cc}
-2 v X & A X-X A^{T} \\
-A X+X A^{T} & -2 v X
\end{array}\right)<0, X>0 .
$$


Нарешті конічному сектору $D_{5}=\left\{z \in C:\{\operatorname{Re}(z) \operatorname{tg} \varphi<\mid \operatorname{Im} z \|\}_{-}\right.$(рис. 1д) відповідає функція:

$$
f_{D_{5}}(z)=\left(\begin{array}{cc}
(z+\bar{z}) \sin \varphi & (z-\bar{z}) \cos \varphi \\
-(z-\bar{z}) \cos \varphi & (z+\bar{z}) \sin \varphi
\end{array}\right)
$$

і лінійні матричні нерівності:

$$
\left(\begin{array}{cc}
\left(A X+X A^{T}\right) \sin \varphi & \left(A X-X A^{T}\right) \cos \varphi \\
-\left(A X-X A^{T}\right) \cos \varphi & \left(A X+X A^{T}\right) \sin \varphi
\end{array}\right)<0, X>0 .
$$

Застосуємо розглянутий апарат для синтезу модального керування лінійної системою для заданої LMI-області. Класичний підхід до синтезу лінійних зворотних зв'язків (регуляторів) в просторі станів пов'язаний з канонічним поданням керованого об'єкта і побудовою модального керування (регулятора), який забезпечує задані власні значення (моди) матриці замкнутої системи. Тоді побудова модального керування зводиться до знаходження характеристичного полінома матриці $A$, вибору канонічного базису і розв'язування системи лінійних рівнянь. Водночас можливий альтернативний шлях синтезу стабілізувальних регуляторів, заснований на застосуванні теорії лінійних матричних нерівностей і ефективних алгоритмів їх розв'язування, реалізованих, наприклад, у пакеті MatLab [6;8].

Нехай об'єкт керування описується рівнянням:

$$
\frac{d x(t)}{d t}=A x(t)+B u(t),
$$

де $x(t) \in R^{n}$ - стан об'єкта; $u(t) \in R^{m}-$ керування.

Завдання полягає у виборі закону керування $u(t)$ з класу лінійних зворотних зв'язків за станом вигляду:

$$
u(t)=K x(t),
$$

де $K$ - матриця параметрів регулятора відповідного порядку, при якому матриця замкненої системи (7), (8) буде $D$-стійкою, тобто всі іï власні значення коренів лежать в заданій LMI-області.

Згідно з теоремою 1 задача $D$-стійкості зводиться до знаходження матриць $X=X^{T}>0$ і $K$, що задовольняють нерівність $M(A+B K, X)<0$, яка $є$ нелінійною відносно цих матриць. Однак, якщо ввести позначення $Z=K X$, то останню нерівність можна уявити як лінійну матричну нерівність вигляду:

$$
\begin{gathered}
M(A+B K, X)=P \otimes X+G \otimes((A+B K) X)+G^{T} \otimes\left(X(A+B K)^{T}\right)= \\
=P \otimes X+G \otimes(A X+B Z)+G^{T} \otimes(A X+B Z)^{T}= \\
=P \otimes X+G \otimes(A X)+G \otimes(B Z)+ \\
+G^{T} \otimes(A X)^{T}+G^{T} \otimes(B Z)^{T}=M(A, X)+G \otimes(B Z)+G^{T} \otimes(B Z)^{T}<0
\end{gathered}
$$

щодо невідомих матриць $K$ і $Z$. Після того, як ці матриці будуть знайдені, шукана матриця параметрів регулятора знаходиться як $K=Z X^{-1}$. 
Розглянемо ще один підхід до синтезу $D$-стабілізувальних регуляторів за вимірюваним виходом, заснований на побудові спостерігачів стану об'єкта. Почнемо з спостерігачів Луенбергера повного порядку.

Для керованого об'єкта:

$$
\left\{\begin{array}{l}
\dot{x}(t)=A x(t)+B u(t), \\
y(t)=C x(t),
\end{array}\right.
$$

де $x(t) \in R^{n}$ - стан регулятора; $u(t) \in R^{m}$ - керування; $y(t) \in R^{p}$ - вимірюваний вихід об'єкта).

Виберемо регулятор у формі спостерігача стану Луенбергера повного порядку:

$$
\left\{\begin{array}{l}
\dot{x}_{r}(t)=A x_{r}(t)+B u(t)+L\left(C x_{r}(t)-y(t)\right), \\
u(t)=K x_{r}(t),
\end{array}\right.
$$

де $x_{r}(t) \in R^{n}$ - стан регулятора.

Необхідно визначити матриці $K$ i $L$ так, щоб замкнута система (9), (10) була $D$-стійкою.

Введемо вектор неузгодженості $e(t)=x(t)-x_{r}(t)$ і як стан замкненої системи виберемо вектор $\left(x^{T}(t), e^{T}(t)\right)^{T}$, який задовольняє узагальнене рівняння:

$$
\frac{d}{d t}\left(\begin{array}{l}
x(t) \\
e(t)
\end{array}\right)=\left(\begin{array}{cc}
A+B K & -B K \\
0 & A+L C
\end{array}\right)\left(\begin{array}{l}
x(t) \\
e(t)
\end{array}\right) .
$$

Очевидно, що для $D$-стійкості цієї системи необхідно і достатньо, щоб матриці $A+B K$ і $A+L C$ були $D$-стійкі. Застосовуючи тепер до матриці $A+B K$ теорему 1 , в якій на мові лінійних матричних нерівностей наведено критерій $D$-стійкості, приходимо до такого вигляду LMI:

$$
\begin{aligned}
M\left(A+B K, X_{1}\right) & =P \otimes X_{1}+G \otimes\left((A+B K) X_{1}\right)+G^{T} \otimes\left(X_{1}(A+B K)^{T}\right)= \\
& =M\left(A, X_{1}\right)+G \otimes\left(B Z_{1}\right)+G^{T} \otimes\left(Z_{1}^{T} B^{T}\right)<0,
\end{aligned}
$$

де $Z_{1}=K X_{1}$.

Застосовуючи до матриці $A+L C$ критерій $D$-стійкості у вигляді нерівності (6), отримаємо ще одну LMI:

$$
\begin{aligned}
L\left(A+L C, X_{2}\right) & =P \otimes X_{2}+G \otimes\left(X_{2}(A+L C)\right)+G^{T} \otimes\left((A+L C)^{T} X_{2}\right)= \\
& =L\left(A, X_{2}\right)+G \otimes\left(Z_{2} C\right)+G^{T} \otimes\left(C^{T} Z_{2}^{T}\right)<0,
\end{aligned}
$$

де $Z_{2}=X_{2} L$.

Таким чином приходимо до необхідності виведення теореми 2.

Для того, щоб об'єкт (9) міг бути D-стабілізованим за допомогою регулятора за виходом виду (10), необхідно і достатньо, щоб лінійні матричні нерівності (11) і (12) були розв'язувані щодо змінних $X_{1}=X_{1}^{T}>0, Z_{1}$ i $X_{2}=X_{2}^{T}>0, Z_{2}$. У разі можливості розв'язання цих нерівностей параметри регулятора знаходяться так:

$$
K=Z_{1} X_{1}^{-1}, L=X_{2}^{-1} Z_{2}
$$


Синтезуємо тепер регулятор на основі спостерігача Луенбергера неповного порядку $[1 ; 5]$. Нехай в об’єкті керування (9) ранг матриці $C$ дорівнює $p$ $(p<n)$. Розглянемо спостерігач

$$
\frac{d z(t)}{d t}=F z(t)+T B u(t)+Q y(t),
$$

де $z(t) \in R^{l} ; l=n-p$ - стан спостерігача; $y(t)$ и $u(t)$ - вимірюваний вихід і керування в об'єкті (9), а матриці $F, T$ i $Q$ задовольняють матричне рівняння

$$
T A-F T=Q C .
$$

Знову введемо вектор неузгодженості $e(t)=z(t)-T x(t)$ i зауважимо, що в силу рівнянь об'єкта і спостерігача для нього виконується рівність:

$$
\frac{d e(t)}{d t}=F e(t) .
$$

Тож якщо матриця $F \in D$-стійкою, то вектор $z(t)$ асимптотично відстежує вектор $T x(t)$ і в сукупності з вектором $y(t)$ дає оцінку вектора стану об'єкта.

Для спрощення, але не зменшуючи спільності, приймемо $C=\left(E_{p} 0_{p} \times\right)$, де $E_{p}$ - одинична матриця розмірності $p$. Зауважимо, що цього можна досягти шляхом відповідної заміни змінних. Розіб'ємо матриці $A$ і $B$ на блоки

$$
A=\left(\begin{array}{ll}
A_{11} & A_{12} \\
A_{21} & A_{22}
\end{array}\right), B=\left(\begin{array}{l}
B_{1} \\
B_{2}
\end{array}\right),
$$

в яких $A_{11} \in R^{p \times p}, B_{1} \in R^{p \times m}-$ (порядки інших блоків визначаються так само).

Виберемо матриці $F, T$ і $Q$, що задовольняють рівняння (14), в такий спосіб

$$
F=A_{22}+L A_{12}, T=\left(\begin{array}{ll}
L & E_{l}
\end{array}\right), Q=A_{21}+L A_{11}-\left(A_{22}+L A_{12}\right) L,
$$

де матрицю $L$ слід визначити з умови, щоб матриця $F$ була $D$-стійкою.

Згідно із зробленим вибором представимо рівняння регулятора (керування) у вигляді:

$$
\begin{gathered}
\frac{d x_{r}(t)}{d t}=\left(A_{22}+L A_{12}\right) x_{r}(t)+\left(B_{2}+L B_{1}\right) u(t)+\left[A_{21}+L A_{11}-\left(A_{22}+L A_{12}\right) L\right] y(t), \\
u(t)=K_{1} x_{r}(t)+K_{2} y(t),
\end{gathered}
$$

де матриці $K_{1}$ i $K_{2}$ належить визначати з умови $D$-стійкості замкненої системи (9), (16). Підставляючи рівняння керування у вихідну систему і враховуючи, що $x_{r}(t)=T x(t)+e(t)$, отримаємо:

$$
\left\{\begin{array}{l}
\frac{d x(t)}{d t}=(A+B K) x(t)-B K_{1} e(t) \\
\frac{d e(t)}{d t}=F e(t)
\end{array},\right.
$$

де $K=\left(\begin{array}{ll}K_{2}+K_{1} L & K_{1}\end{array}\right)$. 
Отже, матриця $K$ знаходиться з умови, щоб матриця $A+B K$ була $D$-стійкою, а потім з урахуванням вже знайденої матриці $L$ визначаються матриці регулятора $K_{1}$ і $K_{2}$. Застосовуючи тепер теорему 1 та техніку виведення теореми 2, приходимо до теореми 3.

Для того, щоб об'єкт, описуваний системою (9), міг бути $D$-стабілізованим за допомогою регулятора за виходом зниженого порядку виду (16), необхідно і достатньо, щоб лінійні матричні нерівності

$$
\begin{gathered}
M\left(A+B K, X_{1}\right)=M\left(A, X_{1}\right)+G \otimes\left(B Z_{1}\right)+G^{T} \otimes\left(Z_{1}^{T} B^{T}\right)<0, \\
L\left(A_{22}+L A_{12}, X_{2}\right)=L\left(A_{22}, X_{2}\right)+G \otimes\left(Z_{2} A_{12}\right)+G^{T} \otimes\left(A_{12}^{T} Z_{2}^{T}\right)<0
\end{gathered}
$$

були розв'язуваними щодо матричних змінних $X_{1}=X_{1}^{T}>0, Z_{1}$ i $X_{2}=X_{2}^{T}>0$, $Z_{2}$, де матриці $M\left(A, X_{1}\right)$ і $L\left(A_{22}, X_{2}\right)$ визначаються за формулами (3) і (6) відповідно. У разі можливості розв'язання цих нерівностей параметри регулятора знаходяться так:

$$
K_{1}=H_{2}, K_{2}=H_{1}-H_{2} L
$$

де

$$
H=\left(\begin{array}{ll}
H_{1} & H_{2}
\end{array}\right)=Z_{1} X_{1}^{-1}, H_{1} \in R^{m \times p}, H_{2} \in R^{m \times l}, L=X_{2}^{-1} Z_{2} .
$$

\section{Висновки}

Отже, застосування спостерігачів Луенбергера дає змогу здійснити синтез $D$-стійких регуляторів щодо виходу спостерігачів Луенбергера повного і зниженого порядку на основі розв'язування тільки лінійних матричних нерівностей.

\section{Лiтература}

1. Баландин Д.В. Синтез законов управления на основе линейных матричных неравенств / Д.В.Баландин, М.М. Коган. — Москва : Физматлит, 2007. — 281 с.

2. Гантмахер Ф.Р. Теория матриц. - Москва : Физматлит, 2004. - 560 с.

3. Поляк Б.Т. Управление линейными системами при внешних возмущениях: Техника линейных матричных неравенств / Б.Т. Поляк, М.В. Хлебников. - Москва : ЛЕНАНД, 2014. $-560 \mathrm{c}$.

4. Якубович B.A. Решение некоторых матричных неравенств, встречающихся в теории автоматического регулирования / ДАН СССР. — 1962. — Т. 143, № 6. — С. 1304-1307.

5. Boyd S., El Ghaoui L., Feron E., Balakrishnan V. LinearMatrix Inequalities in System and Control Theory. — Philadelphia : SIAM, 1994. - $193 \mathrm{p}$.

6. Chilali M., Gahinet P. $H^{\infty}$ design with pole placement constraints: An LMI approach IEEE Trans. Automat. Contr. - 1996. - Vol.41. - P. 358-367.

7. Ghaoui L.E., Niculescu S.I. Advances in linear matrix inequality methods in control. Advances in Design and Control. — Philadelphia, PA : SIAM, 2000. - $372 \mathrm{p}$.

8. Masubuchi I., Ohara A., Suda N. LMI-based controller synthesis: A unified formulation and solution Int. J. Robust Nonlinear Contr.- 1998. — Vol. 8. - P. 669-686. 